\title{
FREE SUB-BANDS OF FINITELY PRESENTED BANDS
}

\author{
JOSEPH LAU \\ School of Mathematics and Statistics, University of Sydney, N.S.W. 2006, Australia
}

(Received 28 April, 1997; revised 12 August, 1997)

\begin{abstract}
For each variety of bands $\mathcal{V}$, we give a formula for $\phi_{\mathcal{V}}(m, k)$, which is the largest integer such that for every band $B$ in $\mathcal{V}$ generated by $m$ generators and $k$ relations, there is a subset of the generators of size $\phi_{\mathcal{V}}(m, k)$ which generates a (relatively) free sub-band of $B$ as a basis. We also determine the semilattice structure of a finitely presented band.
\end{abstract}

1. Introduction and preliminaries. Let $\mathcal{V}$ be a variety of algebras. For integers $m>k$, we define $\phi_{\mathcal{V}}(m, k)$ to be the largest integer such that for every algebra $B$ in $\mathcal{V}$ presented by $m$ generators $a_{1}, \ldots, a_{m}$ and $k$ relations, it is possible to choose $\phi_{\mathcal{V}}(m, k)$ elements from among $a_{1}, \ldots, a_{m}$ which generate a (relatively) free subalgebra of $B$ as a basis.

The question of finding $\phi_{\mathcal{V}}$ for the variety of groups was posed by Lyndon [7, Question 5.29]. Romanovskii [8] showed that $\phi_{\mathcal{V}}(m, k)=m-k$ in this case, but the proof is not constructive. For the variety of semigroups Shneerson [10] gave a constructive proof, based on linear algebra, that $\phi_{\mathcal{V}}(m, k)=m-k$. Standard linear algebra methods, applied to abelian groups (see [5, Chapter 10]), shows that $\phi_{\mathcal{V}}(m, k)=m-k$ for the variety of abelian groups also. This implies, by an easy exercise, that $\phi_{\mathcal{V}}(m, k)=m-k$ for the variety of commutative semigroups also.

Let $\mathcal{V}$ be a variety of algebras. Let $A$ be an alphabet and $R$ a set of relations. Then $\langle A \mid R\rangle_{\mathcal{V}}$ will denote the algebra in $\mathcal{V}$ generated by $A$, subject to the relations of $R$. The subscript $\mathcal{V}$ is sometimes dropped when it is clear from context. We record the following properties of $\phi_{\mathcal{V}}$.

Lemma 1.1. For any variety of algebras $\mathcal{V}$,

(i) $\phi_{\mathcal{V}}\left(m_{1}+m_{2}, k_{1}+k_{2}\right) \leq \phi_{\mathcal{V}}\left(m_{1}, k_{1}\right)+\phi_{\mathcal{V}}\left(m_{2}, k_{2}\right)$, and

(ii) $\phi_{\mathcal{V}}(m, k) \leq \phi_{\mathcal{V}}(m-1, k-1)$.

Proof. If $S_{1}=\left\langle A_{1} \mid R_{1}\right\rangle_{\mathcal{V}}$ and $S_{2}=\left\langle A_{2} \mid R_{2}\right\rangle_{\mathcal{V}}$ are two algebras defined with disjoint alphabets, then $A_{0} \subseteq A_{1} \cup A_{2}$ generates a relatively free subalgebra as a basis in $\left\langle A_{1} \cup A_{2} \mid R_{1} \cup R_{2}\right\rangle_{\mathcal{V}}$ only if $A_{0} \cap A_{i}$ does so in $S_{i}$ for $i=1,2$. Hence (i) follows.

Suppose $S=\langle A \mid R\rangle_{\mathcal{V}}$. Choose $a \in A$ and a letter $b$ not in $A$. We prove (ii) by considering $\langle A, b \mid R, a=b\rangle_{\mathcal{V}}$, using similar reasoning to the above.

In this paper, we give a formula for $\phi_{\mathcal{V}}(m, k)$ for each variety of bands $\mathcal{V}$. Section 2 proves a combinatorial lemma which is the main ingredient of the proof of the formula in Section 3.

We shall use the following notation: the free semigroup over a set $A$ is denoted by $F_{A}$. If $w \in F_{A}{ }^{1}$ we define its content $C(w)$ to be the set of elements of $A$ appearing in $w$. 


\section{A combinatorial lemma.}

Lemma 2.1. Let $A$ be a set of size $m$. Let $A_{1}, A_{2}, \ldots, A_{k}$ be subsets of $A$ such that $\left|A_{i}\right| \geq l$ for each $i$. Then there exists a subset $A^{\prime}$ of $A$ such that

(i) $\left|A^{\prime}\right| \leq \min \left\{\left\lfloor\frac{m+(l-1) k}{2 l-1}\right\rfloor, k\right\}$,

(ii) $A^{\prime} \cap A_{i} \neq \emptyset$ for each $i$.

Proof. Let $n=l k-m$. We shall deal with the case when $n \leq 0$ first, and then prove the lemma for $n>0$ by induction.

If $n \leq 0$, then $m \geq l k$ and $\left\lfloor\frac{m+(l-1) k}{2 l-1}\right\rfloor \geq k$. For each $i$ choose $a_{i} \in A_{i}$. The lemma is proved by letting $A^{\prime}=\left\{a_{1}, a_{2}, \ldots, a_{k}\right\}$.

Now suppose $n>0$. Since $\left|A_{i}\right| \geq l$ for each $i$ and $l k>m$, some $a_{0} \in A$ is in (at least) two of the $A_{i}$ 's. In other words,

$$
k^{\prime}=\left|\left\{i \mid a_{0} \notin A_{i}\right\}\right| \leq k-2 .
$$

Since $l k^{\prime}-(m-1) \leq l k-m-(2 l-1)<n$, by induction there is a subset $B$ of $A \backslash\left\{a_{0}\right\}$ such that

$$
\begin{aligned}
|B| & \leq \min \left\{\left\lfloor\frac{(m-1)+(l-1) k^{\prime}}{2 l-1}\right\rfloor, k^{\prime}\right\} \\
& \leq \min \left\{\left\lfloor\frac{(m+(l-1) k-(2 l-1)}{2 l-1}\right\rfloor, k-2\right\} \\
& \leq \min \left\{\left\lfloor\frac{m+(l-1) k}{2 l-1}\right\rfloor, k\right\}-1,
\end{aligned}
$$

and $B \cap A_{i} \neq \emptyset$ if $a_{0} \notin A_{i}$. The lemma is proved by letting $A^{\prime}=\left\{a_{0}\right\} \cup B$.

Obviously, the bound on $\left|A^{\prime}\right|$ can be attained when $m \geq l k$, and when $m=l$, the bound can be improved to $\left|A^{\prime}\right|=1$. I do not know the necessary and sufficient conditions for the bound in Lemma 2.1 to be sharp.

Using Lemma 2.1 in the case $l=2$ allows us to prove the following combinatorial lemma, crucial to the proof of the formula in Section 3.

Lemma 2.2. Let $A$ be a set of size $m$. Suppose $A_{1}, A_{2}, \ldots, A_{k}$ and $B_{1}, B_{2}, \ldots, B_{k}$ are subsets of $A$ such that for each $i$, either

(a) $\left|A_{i}\right| \geq 2$ and $\left|B_{i}\right| \geq 2$, or

(b) $\left|A_{i}\right|=1$ and $A_{i} \subseteq B_{i}$.

Then there exists a subset $A^{\prime}$ of $A$ such that

(i) $\left|A^{\prime}\right| \leq k+\min \left\{\left\lfloor\frac{m-k}{3}\right\rfloor, k\right\}$,

(ii) $A^{\prime} \cap A_{i} \neq \emptyset$ and $A^{\prime} \cap B_{i} \neq \emptyset$ for each $i$.

Proof. By reordering we can assume $\left|A_{i}\right|=1$ if and only if $i \in\left\{1,2, \ldots, k_{0}\right\}$ for some $k_{0} \leq k$. Let $C=A_{1} \cup A_{2} \cup \cdots \cup A_{k_{0}}$. Then by condition (b),

$$
k^{\prime}=\left|\left\{i \mid C \cap A_{i}=\emptyset\right\}\right|+\left|\left\{i \mid C \cap B_{i}=\emptyset\right\}\right| \leq 2\left(k-k_{0}\right) \leq 2(k-|C|) .
$$

Applying Lemma 2.1 with $l=2$, we obtain a subset $D$ of $A \backslash C$ so that 


$$
\begin{aligned}
|D| & \leq \min \left\{\left\lfloor\frac{(m-|C|)+k^{\prime}}{3}\right\rfloor, k^{\prime}\right\} \\
& \leq \min \left\{\left\lfloor\frac{(m-|C|+2(k-|C|)}{3}\right\rfloor, 2(k-|C|)\right\} \\
& =(k-|C|)+\min \left\{\left\lfloor\frac{(m-|C|)-(k-|C|)}{3}\right\rfloor, k-|C|\right\} \\
& \leq k+\min \left\{\left\lfloor\frac{m-k}{3}\right\rfloor, k\right\}-|C|,
\end{aligned}
$$

$D \cap A_{i} \neq \emptyset$ if $C \cap A_{i}=\emptyset$, and $D \cap B_{i} \neq \emptyset$ if $C \cap B_{i}=\emptyset$. The lemma is proved by letting $A^{\prime}=C \cup D$.

Note that the proofs of the lemmas provide an algorithm for finding the required $A^{\prime}$. The algorithm guarantees an upper bound on the size of $A^{\prime}$, but may not give the smallest possible $A^{\prime}$.

3. The formula. In this section we determine a formula for $\phi_{\mathcal{V}}(m, k)$ for each variety of bands $\mathcal{V}$. We assume familiarity with the basic results from the theory of semigroups and bands, which can be found in [6].

THEOREM 3.1. In any band $B, \mathcal{J}$ equals the minimum semilattice congruence on $B$, and $B$ is a semilattice of rectangular bands.

COROLlary 3.2. Let $T$ be a sub-band of a band $B$. Then for all $x, y$ in $T, x \mathcal{J} y$ in $T$ if and only if $x \mathcal{J} y$ in $B$.

Proof. If $x \mathcal{J} y$ in a band, then $x$ and $y$ lie in the same rectangular band. Therefore $x y x=x$ and $y x y=y$.

The lattice LB of varieties of bands has been determined by Birjukov [1] Fennemore [2] and Gerhard [3] (see also [4]). Only the following fact will be needed in this paper ([9, Remark 3.11]).

Let $\mathbf{L B}_{\mathbf{0}}$ be the lattice obtained from $\mathbf{L B}$ by taking away the four varieties of trivial, right zero, left zero, and rectangular bands. If $\mathcal{V} \in \mathbf{L B}_{\mathbf{0}}$, then for a set $A$, two words in $F_{A}$ represent the same element in the free band on $A$ in $\mathcal{V}$ only if they have the same content.

We first treat the case of the subvarieties of rectangular bands.

THEOREM 3.3. $\phi_{\mathcal{V}}(m, k)=m-k$ for the variety of left [right] zero semigroups. $\phi_{\mathcal{V}}(m-k)=\max \{m-2 k, 1\}$ for the variety of rectangular bands.

Proof. If $w \in F_{A}$, denote its initial and terminal letters by $h(w)$ and $t(w)$ (head and tail) respectively.

Let $\mathcal{L} \mathcal{Z}$ and $\mathcal{R Z}$ denote the varieties of left and right zero semigroups respectively. If $\mathcal{V}=\mathcal{L} \mathcal{Z}$, then

$$
\left\langle A \mid u_{i}=v_{i}, i \in I\right\rangle_{\mathcal{V}} \simeq\left\langle A \mid h\left(u_{i}\right)=h\left(v_{i}\right), i \in I\right\rangle_{\mathcal{V}},
$$

and so the result is clear. The case of right zero semigroups is dual. 
If $\mathcal{V}$ is the variety of rectangular bands, then it is easy to see that

$$
\begin{aligned}
\langle A| u_{i} & \left.=v_{i}, i \in I\right\rangle_{\mathcal{V}} \simeq\left\langle A \mid h\left(u_{i}\right) t\left(u_{i}\right)=h\left(v_{i}\right) t\left(v_{i}\right), i \in I\right\rangle_{\mathcal{V}} \\
& \simeq\left\langle A \mid h\left(u_{i}\right)=h\left(v_{i}\right), i \in I\right\rangle_{\mathcal{L Z}} \times\left\langle A \mid t\left(u_{i}\right)=t\left(v_{i}\right), i \in I\right\rangle_{\mathcal{R Z}}
\end{aligned}
$$

and the result follows.

For the rest of the section let $\mathcal{V}$ be a variety in $\mathbf{L B}_{\mathbf{0}}$.

Let $B$ be a band in the variety $\mathcal{V}$ defined by the presentation

$$
B=\left\langle A \mid u_{i}=v_{i}, i \in I\right\rangle_{\mathcal{V}},
$$

where $I$ is some index set and $u_{i}, v_{i} \in F_{A}$ for each $i \in I$. Let $S L(B)$ be the semilattice defined by the presentation $\left\langle A \mid u_{i}=v_{i}, i \in I\right\rangle$. The $\mathcal{J}$ relation on $B$ is simply denoted by $\mathcal{J}$.

Theorem 3.4. The semilattice $\mathcal{B} / \mathcal{J}$ is isomorphic to $S L(B)$.

Proof. Since $\mathcal{V} \in \mathbf{L B}_{\mathbf{0}}, \mathcal{V}$ contains the variety of semilattices and so the theorem follows from Theorem 3.1.

In particular, if $B$ is the free band on $A$ in $\mathcal{V}$, then $\mathcal{B} / \mathcal{J}$ is isomorphic to the free semilattice on $A$ and so we have the following result.

Corollary 3.5. Suppose that a subset $A_{0}$ of $A$ generates a (relatively) free subband of $B$. Then $A_{0}$ generates a free sub-semilattice of $S L(B)$.

Proof. Let $\phi$ denote the isomorphism from $\mathcal{B} / \mathcal{J}$ to $S L(B)$. Let $T$ denote the subband generated by $A_{0}$, and $\mathcal{J}^{T}$ the $\mathcal{J}$ relation on $T$. Then

$$
\phi(T) \simeq T / \mathcal{J}^{T}
$$

by Corollary 3.2. Since $\phi(T)$ is precisely the sub-semilattice of $S L(B)$ generated by $A_{0}$, the result follows.

Before proving the formula for $\phi_{\mathcal{V}}$ we give an upper bound on $\phi_{\mathcal{V}}(m, k)$ in the case of semilattices.

Lemma 3.6. If $\mathcal{V}$ is the variety of semilattices, then for $m>k$,

$$
\phi_{\mathcal{V}}(m, k) \leq m-\left(k+\min \left\{\left\lfloor\frac{m-k}{3}\right\rfloor, k\right\}\right)
$$

Proof. We first prove a special case, then use it to prove the lemma in general.

Let $E=\langle a, b, c, d \mid a b=c d\rangle_{\mathcal{V}}$. If $B \subset\{a, b, c, d\}$ has 3 elements, then either $\{a, b\} \subset B$ or $\{c, d\} \subset B$. Suppose without loss of generality that $B=\{a, b, c\}$. Since $a b c=(a b)^{2} c=a b c d c=a b c d=a b, B$ does not freely generate a sub-semilattice of $E$. Therefore $\phi_{\mathcal{V}}(4,1) \leq 2$. 
Case 1. Suppose $\left\lfloor\frac{m-k}{3}\right\rfloor \geq k$; that is, $m=4 k+l$ for some $l \geq 0$. Then, by Lemma 1.1 (i),

$$
\phi_{\mathcal{V}}(m, k) \leq \phi_{\mathcal{V}}(l, 0)+\sum_{i=1}^{k} \phi_{\mathcal{V}}(4,1) \leq l+2 k=m-2 k .
$$

Case 2. If $\left\lfloor\frac{m-k}{3}\right\rfloor \leq k$, then writing $m^{\prime}=m-\left(k-\left\lfloor\frac{m-k}{3}\right\rfloor\right)$ and $k^{\prime}=k-\left(k-\left\lfloor\frac{m-k}{3}\right\rfloor\right)$,

$$
\phi_{\mathcal{V}}(m, k) \leq \phi_{\mathcal{V}}\left(m^{\prime}, k^{\prime}\right) \leq m^{\prime}-2 k^{\prime}=m-\left(k+\left\lfloor\frac{m-k}{3}\right\rfloor\right),
$$

where the first inequality follows from Lemma 1.1 (ii), and the second inequality follows from Case 1, because $\left\lfloor\frac{m^{\prime}-k^{\prime}}{3}\right\rfloor=\left\lfloor\frac{m-k}{3}\right\rfloor=k^{\prime}$.

Theorem 3.7. Suppose $\mathcal{V} \in \mathbf{L B}_{\mathbf{0}}$. Then for $m>k$,

$$
\phi_{\mathcal{V}}(m, k)=m-\left(k+\min \left\{\left\lfloor\frac{m-k}{3}\right\rfloor, k\right\}\right) .
$$

Proof. Let $A$ be an alphabet of size $m$ and let

$$
S=\left\langle A \mid u_{1}=v_{1}, u_{2}=v_{2}, \ldots, u_{k}=v_{k}\right\rangle_{\mathcal{V}}
$$

We need to show that there exists a subset $A_{0}$ of $A$ of the correct size that generates a (relatively) free sub-band of $S$ as a basis.

Case 1. Suppose that for each $i$, either

(a) $\left|C\left(u_{i}\right)\right| \geq 2$ and $\mid C\left(v_{i}\right) \geq 2$, or

(b) $\left|C\left(u_{i}\right)\right|=1$ and $C\left(u_{i}\right) \subseteq C\left(v_{i}\right)$.

By Lemma 2.2, we can obtain a subset $A^{\prime}$ of $A$ such that $\left|A^{\prime}\right| \leq k+$ $\min \left\{\left\lfloor\frac{m-k}{3}\right\rfloor, k\right\}$, and if $A_{0}=A \backslash A^{\prime}$, then $u_{i}, v_{i} \notin F_{A_{0}}$ for any $i$. Therefore, as none of the relations can be applied, $A_{0}$ generates a (relatively) free sub-band of $S$ as a basis. (Note that the hypothesis that $\mathcal{V} \in \mathbf{L B}_{\mathbf{0}}$ is used here.)

Case 2. Suppose that one of the relations, say $u_{k}=v_{k}$, is of the form $a_{0}=w$, where $a_{0} \in A \backslash C(w)$. Let $u_{i}^{\prime}\left[v_{i}^{\prime}\right]$ be the word in $F_{A \backslash\left\{a_{0}\right\}}$ obtained by substituting each occurrence of $a_{0}$ in $u_{i}\left[v_{i}\right]$ by $w$. Then it is easy to check that

$$
S \simeq\left\langle A \mid u_{1}^{\prime}=v_{1}^{\prime}, u_{2}^{\prime}=v_{2}^{\prime}, \ldots, u_{k-1}^{\prime}=v_{k-1}^{\prime}, a_{0}=w\right\rangle_{\mathcal{V}} \simeq S^{\prime}
$$

where we put

$$
S^{\prime}=\left\langle A \backslash\left\{a_{0}\right\} \mid u_{1}^{\prime}=v_{1}^{\prime}, u_{2}^{\prime}=v_{2}^{\prime}, \ldots, u_{k-1}^{\prime}=v_{k-1}^{\prime}\right\rangle_{\mathcal{V}}
$$

and $A_{0} \subseteq A \backslash\left\{a_{0}\right\}$ generates a (relatively) free sub-band of $S$ as a basis if it generates a (relatively) free sub-band of $S^{\prime}$ as a basis. Since 


$$
\begin{gathered}
(m-1)-\left((k-1)+\min \left\{\left\lfloor\frac{(m-1)-(k-1)}{3}\right\rfloor, k-1\right\}\right) \\
\geq m-\left(k+\min \left\{\left\lfloor\frac{m-k}{3}\right\rfloor, k\right\}\right),
\end{gathered}
$$

the problem can be reduced to the first case.

Hence we have shown that

$$
\phi_{\mathcal{V}}(m, k) \geq m-\left(k+\min \left\{\left\lfloor\frac{m-k}{3}\right\rfloor, k\right\}\right) .
$$

Lemma 3.6 and Corollary 3.5 show that the reverse inequality also holds. Hence the proof is complete.

Note that the proof of the theorem, combined with the procedure in Section 2, gives a procedure to obtain a subset of $A$ of size $\phi_{\mathcal{V}}(m, k)$ which generates a relatively free sub-band.

\section{REFERENCES}

1. A. P. Birjukov, The lattice of varieties of idempotent semigroups, Algebra i Logika, 9 (1970), 255-273 (Russian); English transl. Algebra and Logic 9 (1970), 153-164.

2. C. F. Fennemore, All varieties of bands, Semigroup Forum 1 (1970), 172-179.

3. J. A. Gerhard, The lattice of equational classes of idempotent semigroups, J. Algebra 15 (1970), 195-224.

4. J. A. Gerhard and M. Petrich, Varieties of bands revisited, Proc. London Math. Soc. 58 (3) (1989), 323-350.

5. B. Hartley and T.O. Hawkes, Rings, modules and linear algebra (Chapman \& Hall, London, 1970).

6. J.M. Howie, An introduction to semigroup theory (Academic Press, London, 1976).

7. The Kourovka notebook, unsolved problems in group theory, 7th augm. ed., Amer. Math. Soc. Trans. (2) 121 (Providence, Rhode Island, 1983).

8. N. S. Romanovskii, Free subgroups of finitely presented groups, Algebra i Logika $\mathbf{1 6}$ (1) (1977), 88-97 (Russian); English transl. Algebra and Logic 16 (1977), 62-68.

9. H. Sezinando, The cardinalities of the Green classes of the free objects in varieties of bands, Semigroup Forum 44 (1992), 164-198.

10. L. M. Shneerson, The free subsemigroups of finitely presented semigroups, Sibirsk. Mat. Zh. 15 (1974), 450-454 (Russian). 